constant. I was concerned with the extent to which folk concepts of mental illness need to be ascertained before embarking on any comparative study involving psychiatric symptoms and/or diagnosis. While it may be an ideal counsel to recommend this groundwork before any comparative psychiatric study is undertaken, it is simply not practical and will not be followed. I introduced my example to suggest that differences in folk concepts of mental illness between native whites of Salford and London are unlikely to be of a magnitude to invalidate the use of the same criteria for psychiatric symptoms and diagnosis across the populations. This strategy does not of course exclude the study of other cultural differences between the two populations.

On another issue, it is Dr Littlewood's perception that I have 'put down' local Yoruba knowledge about smallpox. This is a clear illustration of the different value systems held by anthropologists and medical practitioners (although Dr Littlewood is both, in this instance he is taking an anthropologist's stance). As discussed in my previous letter (Journal, August 1990, 157, 296), an anthropologist is neutral as to whether or not people die of smallpox. His or her professional concern is with the local meaning of rituals and their value to the society that practices them. By contrast, the primary concern of the doctor is the prevention of disease and death, and he or she needs to determine whether a practice/ritual is effective in this respect. This by no means always involves 'putting down' non-Western practices. For over 2000 years Ayurvedic practitioners in India were using an effective antipsychotic agent, Rauwolfia, while doctors in the West were beating psychotic patients, chaining them and, even into the present century, immersing them in cold water. The meaning of these practices/rituals would be of interest to a social anthropologist, whereas the primary concern of a psychiatrist is that these ineffective methods of treatment have been replaced by effective ones, initially Rauwolfia.

Institute of Psychiatry

De Crespigny Park

Denmark Hill

London SE5 8AF

\section{Ethnic minorities and the psychiatric system}

SIR: Although we are somewhat uncertain how to acknowledge Littlewood's assessment of our paper (Journal, March 1990, 156, 373-378) as "not unuseful", we are grateful to him for his less ambiguous critical comments (Journal, September $1990,157,451-452)$. We agree that our study does not address the question of subtle or unconscious racism, but this was not our purpose. One of the starting points for our investigation of police admissions to psychiatric hospitals was to find out how much agreement there was between the policeman and psychiatrist in diagnosing mental illness in emergency referrals. Our results revealed a very high degree of concordance. One of the reasons that we undertook this investigation was in response to an assertion, supported by Dr Littlewood, that "the police are overtly racist and selectively pick out nonmentally ill black people in the streets and take them to a psychiatric hospital under Section 136 of the Mental Health Act as an alternative to arrest" (Littlewood, 1986). We believe that our study, carried out in an area with one of the highest Section 136 admission rates in the UK, challenges this assertion, and raises the concern that overt racism is more likely to result in mentally ill subjects from the ethnic minorities being channelled into the criminal justice system instead of the psychiatric services.

We appreciate the value of case vignette studies of the type advocated by Dr Littlewood and used in the study by Lewis et al (Journal, March 1990, 157, 410-415). However, we are concerned that such an enlightened approach should not preclude descriptive studies which detail the progress of the ethnic minorities through the psychiatric services and the criminal justice system. It could even be argued that the results of such an approach (e.g. Harrison et al, 1988), which undoubtedly appear naive to the anthropologist, have brought transcultural psychiatry research to life in this country.

Institute of Psychiatry

THOMAS FAHY

De Crespigny Park

Denmark Hill

London SE5 8AF

Maudsley Hospital

Denmark Hill

London SE5 8AZ

\section{References}

Harrison, G., OWEns, D. \& Holton, A., et al (1988) A prospective study of severe mental disorder in Afro-Caribbean patients. Psychological Medicine, 18, 643-657.

LITTLEWOOD, R. (1986) Ethnic minorities and the Mental Health Act. Bulletin of the Royal College of Psychiatrists, 10, 306-308.

\section{Benzodiazepine withdrawal}

SIR: We thank Hawley for his kind comments on our paper (Journal, November 1990, 157, 777-778) and welcome the opportunity of replying. 
Ideally, we should have liked to have included four treatment groups in our study: abrupt and gradual withdrawal, each with and without propranolol. Such a study was, however, impractical for us at the time. We therefore restricted it to the two groups described in our paper, as these seemed to be the most widely used and effective treatment regimes at the time the study was launched. We agree that our study does not dismiss the possibility of propranolol being of some use in benzodiazepine withdrawal in some patients. However, it is of note that in our sample, patients slowly withdrawn were successful in their efforts at withdrawal without suffering significant withdrawal symptoms. In the slow withdrawal group, therefore, few patients would have needed any adjunct to the slow withdrawal regime. It is also notable that in the abrupt withdrawal group, the addition of propranolol did not prevent patients from suffering considerable withdrawal symptoms that in many cases led to failure to complete the withdrawal process.

We intended to determine whether the advantages of slow withdrawal could be offset by using propranolol, thus allowing abrupt withdrawal to be successful in a similar proportion of cases. We believe that we have shown that this is not the case.

TIM CANTOPHER

Nancy Cleave

GuY EDWARDS

S. OLIVIERI

Abraham Cowley Unit
St Peters Hospital
Guildford Road
Chertsey, Surrey

\section{Limitations of double-blind trials}

SIR: Double (Journal, August 1990, 157, 300) appears to have misunderstood the argument I put forward (Journal, February 1990, 156, 282). The valuable article by Kramer \& Shapiro (1984) he cites does not denigrate the value of the randomised controlled trial, but indeed upholds it as the method of choice for evaluation of therapeutic interventions, although pointing out several issues that are important to the design, execution and interpretation of a clinical trial. One of these issues is the possibility of bias arising when the desirable property of double-blindness is not attained, a possibility I certainly accept as impairing the interpretation of the results of a study.

Kramer \& Shapiro suggested a strategy of asking participants to guess, on completion of the trial, which treatment they received, but solely as a means of assessing possible bias due to unblinding. They explicitly upheld the principle of analysis by 'intention to treat' and did not advocate stratification of the statistical analysis by blinding status. In a trial of ascorbic acid for the common cold, cited both by Dr Double and by Kramer \& Shapiro, Karlowski et al (1975), having found that many more participants guessed their treatment correctly than guessed incorrectly, went on to examine evidence for efficacy in two subgroups separately, those who 'knew' which treatment they had received and those who 'did not know'. These subgroup labels were in fact misleading: the 'knew' group consisted of 79 subjects who guessed correctly; the 'did not know' group comprised not only 88 who did not offer a guess but also 23 who guessed incorrectly. This suggests that some 23 of the 79 who guessed correctly were doing no more than guessing; the 'did not know' group would more appropriately be supplemented by transferring to it 23 of the 'knew' group, either chosen randomly or by a weighted analysis.

Failure to demonstrate an advantage for active treatment in the 'did not know' group, as occurred in Karlowski et al's study, need not imply that the benefit observed in an intention-to-treat analysis was illusory, for such a failure can arise in three ways. Firstly, the 'did not know' group may not be defined symmetrically (i.e. to contain as many correct guesses as incorrect ones). Karlowski et al failed in this respect, and the recommendations of Oxtoby et al (1989) seem to suggest doing just what Karlowski et al did. Secondly, problems can result from predominantly correct guessing, arising because of therapeutic efficacy. Karlowski et al were not able to exclude this and Oxtoby et al would be careful to distinguish it from guessing on the basis of sideeffects. Thirdly, failure can arise from the depletion of the number of subjects included, and consequently of the statistical power to demonstrate a difference in effectiveness between treatments. This can seriously impair ones ability to demonstrate a null difference, as well as one's ability to demonstrate a substantial one.

The implication is clearly that the issue of maintenance of blindness should be dealt with as far as possible by good study design and execution. This will not always avoid the problem, but when it does, it will be much more satisfactory than a somewhat artificial salvaging operation in the statistical analysis. Often the results of a trial will fail to be as definitive as one would like, but will generally be subject to fewer limitations than inferences from purely observational studies. Interpretation of results is inevitably of the greatest importance; equally inevitably, the 